Annals of Pure and Applied Mathematics

Vol. 16, No. 1, 2018, 53-61

ISSN: 2279-087X (P), 2279-0888(online)

Published on 1 January 2018

www.researchmathsci.org

DOI: http://dx.doi.org/10.22457/apam.v16n1a7

Annals of

Pure and Applied

Mathematics

\title{
Mathematical Aspects of Laguerre Wavelets Transformation
}

\author{
Satyanarayana Bavanari and Asma Abdulelah Abdulrahman \\ Department of Mathematics, Acharya Nagarjuna University \\ Nagarjuna Nagar - 522 510, Andhra Pradesh, India. \\ E-mail: drbsn63@yahoo.co.in asmaa a30@yahoo.com
}

Received 5 November 2017; accepted 3 December 2017

Abstract. In this work, many important theorems will be proved and proven in the formation of (DLWT) in that they take their role in the processing of the signal or image processing, which makes them possess characteristics such as those characterized by those that were used for the same purpose and with high efficiency.

Keywords: Laguerre wavelets transform, Laguerre's Scalar function, Multiresolution Analysis of Laguerre wavelets

\section{AMS Mathematics Subject Classification (2010): 42C40}

\section{Introduction}

It is worth mentioning that the analysis of data from the signals or images is done by wavelets in the decisions as we know that wavelet algorithms that deal with signals are working in the same way as the human eye or camera and capture signals cell phone and even digital color images used in medicine are all processed by waveforms as approximation of data and intermittent signals or images with too many edges $[1,9,10]$. The result of algorithms is the key to processing numbers or digital information, signals, images etc, [2]. The result of algorithms is a key to processing numbers or digital information, signals and images. Wavelet applications are data compression, fingerprints and many fields of science and engineering [5, 7]. Wavelet image processing enables computers to store an image in many scales of resolutions. Consequently, decomposing an image into various levels and types of approximations with different valued resolutions [3]. Wavelets allow one to compress the image using less storage space with more details of the image [4].

\section{Laguerre wavelets}

In this section authors will construct Laguerre wavelet from the family function $[6,8]$.

$$
\rho_{\mathrm{s}, \mathrm{r}}(\mathrm{t})=|\mathrm{s}|^{-1 / 2} \rho\left(\frac{\mathrm{t}-\mathrm{r}}{\mathrm{s}}\right), \forall \mathrm{s}, \mathrm{r} \in \mathrm{R}, \mathrm{s} \neq 0
$$

where $\rho(t)=\left[\rho_{0}(t), \rho_{1}(t), \ldots, \rho_{M-1}\right]^{T}$. 
Satyanarayana Bavanari and Asma Abdulelah Abdulrahman

The elements $\rho_{0}(t), \rho_{1}(t), \ldots, \rho_{M-1}(t)$ are the basis functions, orthogonal in the interval $[0,1]$.

Laguerre wavelet is denoted by $\operatorname{Lag}($ wav $)$, is the type of wavelets and is used for solving differential equation, integral equation, variation problems and different sciences and engineering as well as fractional differential equations. That is why it is an important part. Laguerre wavelet

$$
\rho_{\mathrm{n}, \mathrm{m}}(\mathrm{t})=\rho_{\mathrm{t}, \mathrm{n}, \mathrm{m}, \mathrm{k}}
$$

have four arguments $\mathrm{k}=1,2, \ldots, \mathrm{n}=1,2, \ldots, 2^{\mathrm{k}-1}, \mathrm{~m}$ is order for Laguerre polynomials and $t$ is normalized time. If we dilation by parameter $s=2^{-(k+1)}$ and translation by parameter $\mathrm{r}=2^{-(\mathrm{k}+1)}(2 \mathrm{n}-1)$ by transform $\mathrm{x}$ and by using (1).

If $x=2^{-(k+1)}\left(2^{k} t\right)$ then we will get the following equation

$$
\rho_{\mathrm{n}, \mathrm{m}}(\mathrm{t})=\left\{\begin{array}{lc}
2^{\frac{\mathrm{k}+1}{2}} \tilde{\mathrm{L}}_{\mathrm{m}}\left(2^{\mathrm{k}} \mathrm{t}-2 \mathrm{n}+1\right), & \frac{\mathrm{n}-1}{2^{\mathrm{k}-1}} \leq \mathrm{t} \leq \frac{\mathrm{n}}{2^{\mathrm{k}-1}} \\
0 \quad, & \text { otherwise }
\end{array}\right.
$$

where $\tilde{\mathrm{L}}_{\mathrm{m}}=\frac{1}{\mathrm{~m} !} \mathrm{L}_{\mathrm{m}}$, for $\mathrm{k}=2$.

A function approximation $\mathrm{f}(\mathrm{t}) \in \mathrm{L}^{2}[0,1]$ may be expanded as:

$$
f(t)=\sum_{n=1}^{\infty} \sum_{m=0}^{\infty} A_{n, m} \rho_{n, m}(t)
$$

where, $A_{n, m}=\left\langle f(t), \rho_{n, m}(t)\right\rangle$

In equation (4), $\langle.,$.$\rangle denote the inner product with weight function w_{n}(t)$ on the Hilbert Space $[1,0)$. If the infinite series in above equation is truncated, then equation (3) can be written as:

$$
f(t)=\sum_{n=1}^{2^{k-1}} \sum_{m=0}^{M-1} A_{n, m} \rho_{n, m}(t)=A^{T} \rho_{n, m}(t)
$$

where $A$ and $\rho(t)$ are $2^{k-1} M \times 1$ matrices given by:

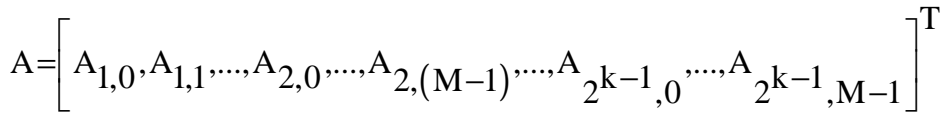

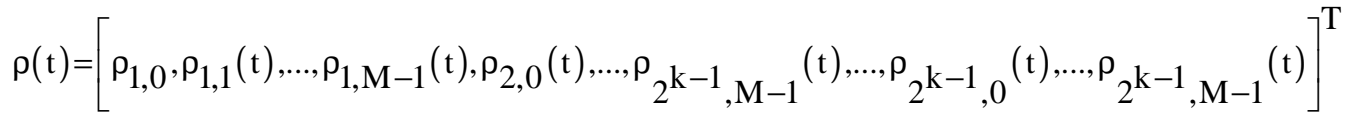




\section{Mathematical Aspects of Laguerre Wavelets Transformation}

\section{Properties of Laguerre wavelets transform}

Forward Wavelet Transform, in addition to the use of waveforms mentioned in many sources, which have many common characteristics that qualify for the operations of the pressure of the image of these qualities such as symmetric and orthogonal. In addition to converting the waveform that we have constructed in the previous chapters and the qualities it possesses, it also gives us the assurance that these waveguides possess the same image processing behavior as the previously used waveforms, for example Haar wavelets transform.

Definition 3.1. For every pair of $n, m \in Z$, define the period $I_{n, m}$ by

$$
I_{n, m}=\left[2^{-n} m, 2^{-n}(m+1)\right]
$$

which is familiar as dyadic period. The group of all such periods is called dyadic sub periods of $R$.

\section{Laguerre's Scalar function}

The family of functions [12],

$$
\left\{\vartheta_{n, m}(t)\right\}_{n, m \in Z}=2^{\frac{-n}{2}} \vartheta\left(2^{-n} t-m\right), \forall n, m \in Z
$$

is called the system of Laguerre Scalar function at scale $n$.

Laguerre Scalar function can be defined as

$$
\vartheta(t)= \begin{cases}1 & , 0 \leq t \leq 1 \\ 0 & , \text { otherwise }\end{cases}
$$

Equation (8) will be

$$
\begin{aligned}
& \mathrm{D}_{2^{\mathrm{n}}} \mathrm{L}_{\mathrm{m}} \vartheta(\mathrm{t}) \\
& D_{r} f(t)=r^{1 / 2} f(r t)
\end{aligned}
$$

And the extending of employee $L_{m} f(t)=f(t-m)$.

\section{The system of Laguerre wavelets LWS}

Laguerre wavelets system for each $\mathrm{n}, \mathrm{m} \in \mathrm{Z}$, defined as

$$
\left\{\rho_{n, m}(t)\right\}_{n, m \in Z}=2^{\frac{-n}{2}} \rho\left(2^{-n} t-m\right), \quad \forall n, m \in Z
$$

From the equation (10), the function is called the Laguerre wavelet system denoted by (LWS). Consider $\mathrm{f}(\mathrm{t})$ is defined on $\mathrm{L}^{2}[0,1]$ has an expansion in terms of Laguerre functions as follows:

For any integer $\geq 0$.

$$
f(t)=\sum_{m=0}^{2^{n}-1}\left\langle f, \vartheta_{N, m}\right\rangle \vartheta_{N, m}(t)+\sum_{n=N}^{\infty} \sum_{m=0}^{2^{n}-1}\left\langle f, \rho_{N, m}\right\rangle \rho_{N, m}(t)
$$


Satyanarayana Bavanari and Asma Abdulelah Abdulrahman

$$
=\sum_{m=0}^{2^{n}-1} a_{n, m} \vartheta_{N, m}(t)+\sum_{n=N}^{\infty} \sum_{m=0}^{2^{n}-1} d_{n, m} \rho_{N, m}
$$

which is known as Laguerre series where $d_{m, n}$ and $a_{n, m}$ are Laguerre coefficients for wavelet and Laguerre scaling coefficients respectively.

\section{Wavelets}

In many sources you can take the basic idea of transforming the wavelet, which is summarized in the following definition. They are functions defined over finite period with average value of zero and for any arbitrary function $f(t)$ as an overlap of a basis functions. It is called the mother wavelet $\rho(t)$, from equation (1). Throughout the study of wavelets they are shown to be the best, most efficient and flexible because of their method as smooth. For each $n, m \in Z$, define $\rho_{n, m}(t)$.

$$
\rho_{\mathrm{n}, \mathrm{m}}(\mathrm{t})=\frac{1}{2^{\mathrm{n}}} \rho\left(2^{-\mathrm{n}} \mathrm{t}-\mathrm{m}\right)
$$

The framework for constructing wavelets involves the concept of a multiresolution analysis of Laguerre wavelets. They are constructed by $\left\{\rho_{n, m}(t)\right\}$, where $n, m \in Z \rightarrow \rho(t) \in L^{2}$ orthonormal on R. Multiresolution analysis of Laguerre wavelets $\left(\mathrm{MRA}_{\mathrm{Lag}(\text { way })}\right)$ is a system for calculation of basis coefficients in

$$
\begin{aligned}
& \mathrm{L}^{2}(\mathrm{R}): \mathrm{f}=\sum \sum \mathrm{A}_{\mathrm{n}, \mathrm{m}} \rho_{\mathrm{n}, \mathrm{m}} \\
& f \in V_{n}=\left\{f(t) / f(t)=\frac{1}{\frac{n}{2}} h\left(2^{-n} t\right), h(t) \in V_{0}\right\},
\end{aligned}
$$

where

$$
f(t)=\sum_{m \in Z}\langle f, \vartheta(\cdot-m)\rangle \vartheta(t-m)
$$

Then a multi resolution analysis of Laguerre wavelets $\left(\mathrm{MRA}_{\mathrm{Lag}(\mathrm{wav})}\right)$ on $\mathrm{R}$ is a sequence of subspaces $\left\{V_{n}\right\}, n \in Z$ of functions $L^{2}$ on R. First and foremost, we should look forward to achieve the following characteristics that allow us to complete our work in the field that authors will apply [3].

(a) $\forall \mathrm{n}, \mathrm{m} \in \mathrm{Z}, \mathrm{V}_{\mathrm{n}} \subseteq \mathrm{V}_{\mathrm{n}+1}$.

(b) If $\mathrm{f}(\mathrm{t})$ is $\mathrm{C}_{\mathrm{c}}^{0}$ on $\mathrm{R}$, then $f(t) \in \operatorname{span}\left\{V_{n}\right\}, n \in Z$, with $\in>0$, there is an $\mathrm{n} \in \mathrm{Z}$ and a function $\mathrm{g}(\mathrm{t}) \in \mathrm{V}_{\mathrm{n}}$ such that $\|\mathrm{f}-\mathrm{g}\|_{2}<\epsilon$.

(c) $\bigcap_{n \in Z} V_{n}=\{0\}$.

(d) A function $f(t) \in V_{0}$ if and only if $2^{-n / 2} f\left(2^{-n} t\right) \in V_{n}$. 
Mathematical Aspects of Laguerre Wavelets Transformation

(e) There exists a function $\vartheta(t), L^{2}$ on $\mathrm{R}$, called the scaling function such that the collection $\vartheta(t-n)$ is an orthonormal system of translates and $V_{0}=\operatorname{span}\{\vartheta(t-n)\}$.

Definition 5.1. Let $\left\{V_{N}\right\}$ be an $\mathrm{MRA}_{\mathrm{Lag}(\mathrm{wav})}$ with calibration function $\vartheta(\mathrm{t})$ which satisfies (13) and $\mathrm{h}(\mathrm{m})$. In this definition authors are considered calibration colander, where

$$
\mathrm{h}(\mathrm{m})=<\frac{1}{\sqrt{2}} \vartheta\left(\frac{\mathrm{t}}{2}\right), \vartheta(\mathrm{t}-\mathrm{m})>
$$

Then, find out the following function which will be named as Wavelet colander $\mathrm{g}(\mathrm{m})$ defined as

$$
\mathrm{g}(\mathrm{t})=(-1)^{\mathrm{m}} \mathrm{h}(1-\mathrm{m})
$$

and the Laguerre wavelet will be defined as

$$
\rho(x)=\sum_{m \in Z} g(m) \frac{1}{\sqrt{2}} \vartheta\left(\frac{t}{2}-m\right)
$$

Then $\left\{\rho_{n, m}(t)\right\} n, m \in Z$ is a Lag wavelet orthonormal standard on $R$.

Definition 5.2. Let $f$ be the arbitrary function then the orthogonal projection of it is given by

$$
\rho_{n} f=\sum_{m \in Z}\left\langle f, \vartheta_{n, m}\right\rangle \vartheta_{n, m}
$$

$\mathrm{f} \in \mathrm{L}^{2}$ when it on to $V_{\mathrm{n}}$.

\section{Approximation by (DLWT) in different Space}

In this section, we prove waveforms belong and are realized approximation space.

\subsection{Demonstrate of approximate space}

1. ( $\operatorname{Lag}($ wav $\left.),\|\cdot\|_{\text {Lag }}\right)$ belong to norm space denoted by $\left(\mathrm{W},\|\cdot\|_{\mathrm{W}}\right)$.

2. Let $\left((\mathrm{Wav})_{\mathrm{N}}\right)_{\mathrm{N} \geq 0}$ be a functions of subspaces of a space $\mathrm{W}$ and $\left((\mathrm{Wav})_{\mathrm{N}}\right)_{\mathrm{N} \geq 0} \subseteq \mathrm{W}$.

3. If $\mathrm{f} \in \mathrm{W}$, ( $\mathrm{f}$ is a function) $\rightarrow \mathrm{E}_{\mathrm{N}}(\mathrm{f})_{\mathrm{w}}=\|\mathrm{f}-\mathrm{K}\|_{\mathrm{w}}$, the approach error, where $\mathrm{K}$ approach in $\left((\mathrm{Wav})_{\mathrm{N}}\right)_{\mathrm{N} \geq 0}$.

\section{Case 1.}

For linear approach, $\mathrm{N}$ perform prerequisite to characterize on element in $W_{N}$. That is, $\mathrm{N}$ is measure of $W_{N}$. There are situations that must take seriously as

$$
\mathrm{N} \rightarrow \infty \text { when } E_{N}(f)_{w} \rightarrow 0 \text {. }
$$

Case 2.

For the nonlinear approach, different from the first case where $\mathrm{N}$ is Linked to free factors. This can be demonstrated by example, $\mathrm{N}$ power be the number of contract in piecewise stationary approximation with free knots $\mathrm{W}_{\mathrm{N}}$ is general space can be quite. Specially, this means they are not linear. 
Satyanarayana Bavanari and Asma Abdulelah Abdulrahman

\subsection{Approximation in Space of square integrable functions over $\mathbf{R}$}

1. Let $f \in L_{2}(R)$ then the $(L a g)_{\text {wav }}$ be a series

$$
f=\sum_{n=0}^{\infty} \sum_{m=0}^{N-1}\left\langle f, \rho_{n, m}\right\rangle \rho_{n, m}(t)
$$

a continuous function.

2. $\rho_{n, m}(t)$ is support on the period

$$
\begin{aligned}
& \mathrm{I}_{\mathrm{n}, \mathrm{m}}=\left[2^{-\mathrm{n}} \mathrm{m}, 2^{-\mathrm{n}}(\mathrm{m}+1)\right] \\
& <f, \rho_{n, m}>=\int f(t) \rho_{n, m}(t) d t=2^{\frac{-n}{2}} \int_{m 2^{-n}}^{(m+1) 2^{-n}} f(t) \rho\left(2^{-n} t-m\right) d t
\end{aligned}
$$

3. If we calculate finite aggregate, we can find the coefficients for some $1 \in \mathrm{N}$ by $\mathrm{N}=2^{\mathrm{I}}, \mathrm{n}=0,1,2, \ldots, 2^{\mathrm{I}}-1=\mathrm{N}$ then

$$
\sum_{\mathrm{n}=0}^{\mathrm{I}-1} \sum_{\mathrm{m}=0}^{\mathrm{N}-1} 1=1+2+2^{2}+\ldots+2^{\mathrm{I}}-1=\mathrm{N}-1
$$

Coefficients, for DLWT we can see that for each n, only one of the coefficients is non zero and its size is $2^{\frac{-n}{2}}$. By using the following equation, we can determine the approximate error in $\mathrm{L}_{2}(\mathrm{R})$ which is denoted by $\mathrm{E}$

$$
\begin{array}{r}
\left\|f-\sum_{n=0}^{I-1} \sum_{m=0}^{N-1}<f, \rho_{n, m}>\rho_{n, m}(t)\right\|_{L_{2}}^{2}=\left\|\sum_{n=I}^{\infty} \sum_{m=0}^{N-1}<f, \rho_{n, m}(t)>\rho_{n, m}(t)\right\|_{L_{2}}^{2} \\
=\sum_{N=1}^{\infty} \sum_{m=0}^{N-1} k f, \rho_{n, m}>\left.\right|^{2}=\sum_{n=1}^{\infty} 2^{-n}=2^{-1}=\frac{1}{N}=\mu\left(2^{-n}\right),
\end{array}
$$

where, $\mu$ is any approximation result.

Theorem 6.3. (Approximation in $L_{P}(R)$ ).

Let $f \in L_{P}(R)$ be the partial sum of (DLWS) of $f$ then

$$
H=\sum_{n=0}^{l-1} \sum_{m=0}^{N-1}\left\langle f, \rho_{n, m}\right\rangle \rho_{n, m}(t) n \in N \rightarrow E_{a p p}=\mu\left(2^{\frac{-n}{2}}\right)
$$

\section{Proof:}

$\mathrm{E}_{\text {app }}$, which determine the approximate error in $\mathrm{L}_{\mathrm{P}}(\mathrm{R})$.

$$
\mathrm{E}_{\text {app }}=\|\mathrm{f}-\mathrm{H}\|_{\mathrm{Lp}}\left\|\mathrm{f}-\sum_{\mathrm{n}=0 \mathrm{~m}=0}^{\mathrm{I}-1} \sum_{\mathrm{m}=0}^{\mathrm{N}-1}<\mathrm{f}, \rho_{\mathrm{n}, \mathrm{m}}>\rho_{\mathrm{n}, \mathrm{m}}(\mathrm{t})\right\|_{\mathrm{Lp}}
$$


Mathematical Aspects of Laguerre Wavelets Transformation

$$
\begin{aligned}
& =\left\|\sum_{n=1}^{\infty} \sum_{m=0}^{N-1}\left\langle f, \rho_{n, m}\right\rangle \rho_{n, m}(t)\right\|_{L_{p}} \\
& =\left(\sum_{n=1}^{\infty} \sum_{m=0}^{N-1}\left\langle\left.\left\langle f, \rho_{n, m}\right\rangle\right|^{p}\right)^{\frac{1}{p}}\right. \\
& =\left(\sum_{n=1}^{\infty} 2^{\frac{-l p}{2}}\right)^{\frac{1}{p}}=2^{\frac{-1}{2}}=\mu 2^{\frac{-1}{2}} .
\end{aligned}
$$

Theorem 6.4. (Approximation in $\mathrm{L}_{\mathrm{iPM}}(\delta, \mathrm{P})$ ).

Let $f \in L_{i p M}(\delta, p), \delta \in(0,1], p \in(0, \infty], M \geq 0$.

Then $H(t)=\sum_{n=0}^{l-1} \sum_{m=0}^{N-1}\left\langle f, \rho_{n, m}\right\rangle \rho_{n, m}(t)$ is the LWS of $f$ for some $\mathrm{I} \in \mathrm{N}$, $E_{\text {app }}=\mu\left(2^{-l \delta}\right)$.

Proof. $\mathrm{E}_{\text {app }}$ is the approximate error of in $\mathrm{F}^{\mathrm{s}} \mathrm{L}_{2}(\mathrm{R})$.

$$
\begin{aligned}
E_{a p p} & =\|f-H\|_{L_{2}}=\left\|f-\sum_{n=0}^{l-1} \sum_{m=0}^{N-1}\left\langle f, \rho_{n, m}\right\rangle \rho_{n, m}(t)\right\| \\
& \leq\left(\sum_{n=l}^{\infty} \sum_{m=0}^{N-1}\left|\left\langle f, \rho_{n, m}\right\rangle\right|^{2}\right)^{\frac{1}{2}}=\left(\sum_{n=l}^{\infty} \sum_{m=0}^{N-1} \frac{2^{s m}}{2^{s N}}\left|\left\langle f, \rho_{n, m}\right\rangle\right|^{2}\right)^{\frac{1}{2}} \\
& =\left(2^{-s N} \sum_{n=l}^{\infty} \sum_{m=0}^{N-1} 2^{s m}\left|\left\langle f, \rho_{n, m}\right\rangle\right|^{2}\right)^{\frac{1}{2}}
\end{aligned}
$$

from spaces' properties $\|f\|_{F^{s}}\left(L_{2}(R)\right) \cong\left(\left(\sum_{n=l}^{\infty} \sum_{m=0}^{N-1} 2^{s m}\left|\left\langle f, \rho_{n, m}\right\rangle\right|^{2}\right)^{\frac{1}{2}}\right)$.

Therefore $E_{\text {app }} \leq 2^{\frac{-S N}{2}}\|f\|_{F^{s}}\left(L_{2}(R)\right)=\mu 2^{\frac{-S N}{2}}$.

Theorem 6.5. (Approximation in Besov Space $B_{r}^{\delta}\left(L_{P}(R)\right)$.

Let $\mathrm{f} \in \mathrm{B}_{\gamma}^{\delta}\left(\mathrm{L}_{\mathrm{P}}(\mathrm{R})\right) \delta>0,0<\gamma \leq \infty$ and $H(t)=\sum_{n=0}^{l-1} \sum_{m=0}^{N-1}\left\langle f, \rho_{n, m}\right\rangle \rho_{n, m}(t)$, can be limited

LWS of $f$. Then there exists $\mathrm{I} \in \mathrm{N} I \in N \rightarrow E_{a p p}=\mu 2^{\frac{-\delta N}{2}}$, where $\mathrm{N}=2^{\mathrm{I}}$. 
Satyanarayana Bavanari and Asma Abdulelah Abdulrahman

Proof is straight forward.

\section{Non linear approximation in $\mathrm{L}_{\mathrm{P}}(\mathrm{R})$.}

In this section we prove only one theorem.

Theorem 7.1. $f \in L_{P}(R)$ the partial sum of LWS $f$ is

$$
H(t)=\sum_{n=0}^{l-1} \sum_{m=0}^{N-1}\left\langle f, \rho_{n, m}\right\rangle \rho_{n, m}(t), n \in N, \rightarrow E_{a p p N L}=\mu\left(2^{\frac{-n}{2}}\right) \text {. }
$$

Proof:

$$
\begin{aligned}
\mathrm{E}_{\text {appNL}}\|\mathrm{f}-\mathrm{H}\|_{\mathrm{L}_{\mathrm{P}}} & =\left\|\mathrm{f}-\sum_{\mathrm{n}=0}^{\mathrm{I}-1} \sum_{\mathrm{m}=0}^{\mathrm{N}-1}<\mathrm{f}, \rho_{\mathrm{n}, \mathrm{m}}>\rho_{\mathrm{n}, \mathrm{m}}(\mathrm{t})\right\|_{\mathrm{L}_{\mathrm{P}}} \\
& =\left\|\sum_{n=0}^{\infty} \sum_{m=0}^{N-1}\left\langle f, \rho_{n, m}\right\rangle \rho_{n, m}(t)\right\|_{L_{p}}=\left(\sum_{n=l+1}^{\infty} \sum_{m=0}^{N-1}\left|\left\langle f, \rho_{n, m}\right\rangle\right|^{p}\right)^{\frac{1}{p}} \\
& =\left(\sum_{n=l+1}^{\infty} 2^{\frac{-l p}{2}}\right)^{\frac{1}{p}}=2^{\frac{-1}{2}}=\mu 2^{\frac{-1}{2}}
\end{aligned}
$$

8. Conclusion.

In this article we have demonstrated many theories that show the mathematical aspects of the Laguerre wavelets where we were able to reach the important definitions that play a very important role in the applications in image processing.

Acknowledgement. The authors are greatly indebted to the reviewer and Editor of the journal for their valuable suggestions to improve the research paper.

\section{REFERENCES}

1. A.M.Raid and W.M.Khedr, JPEG image compression using discrete cosine transform, a survey, International Journal of Computer Sciences \& Engineering Survey, 5(2) (2014) 39-47.

2. Er.Kirandeep Kaur \& Er.Rishma Chawla 'Image Compression Using Wavelet Transform, International Journal of Information Technology and Knowledge Management, 5(2) (2012) 221- 423.

3. M.R.Islam, 'Comparison of wavelet approximation order in different smoothness spaces, International Journal of Mathematics and Mathematical Sciences, 2006 (63670) (2006) 1-7.

4. Othman Khalifa, Wavelet coding design for image data compression, International Arab journal of Information Technology, 2(2) (2005) 118-128. 
Mathematical Aspects of Laguerre Wavelets Transformation

5. Sarvarinder Singh, Er. Sarabjil Singh, Rio based medical image compression using DCT and Haar Wavelet, International Journal of Advanced Research in Computer and Communication Engineering, 4 (2015) 469-472.

6. Sadiq Jassim Abou-Loukh, Jaleel Sadoon Jameel, Compression of an ECG signal using mixed transforms, Journal of Engineering, 20(6) (2014) 109-123.

7. S.Sindhu, V.Geetha and G.Murugesan, Two dimensional dual-mode lifting based discrete wavelet transform, International Journal of Innovative Research in Science, Engineering Technology, 3 (2014) 1558-1563.

8. Tim Bruylants, Adrian Munteanu, Wavelet based volumetric medical image compression, Signal Processing: Image Communication, 31 (2015) 112-133.

9. V.Alarcon-Aquino, J.M.Ramirez-Cortes, Lossy image compression using discrete wavelet transform and thersholding techniques, The open Cybernetics \& systemic Journal, 7(2013) 32-38.

10. V.Ashok, T.Bala kumaran, The fast wavelet transform for signal and image processing, International Journal of Computer Science and Information Security, 7(1) (2010) $126-130$. 